\title{
ON EXTENDING MAPPINGS INTO NONLOCALLY CONVEX LINEAR METRIC SPACES
}

\author{
T. DOBROWOLSKI
}

\begin{abstract}
It is proved that the following spaces are absolute retracts: every $F$-space with a Schauder basis and certain function spaces along with their subgroups of integer-valued elements. It is also observed that for every $\sigma$-compact convex set, the absolute extension property for compacta implies the AR-property.
\end{abstract}

1. Introduction. The purpose of this paper is to provide new examples of infinite-dimensional ANRs. Detecting the ANR-property of convex subsets of nonlocally convex metric linear spaces and topological groups is of great importance. For example, the topological classification of these spaces, given recently in $[\mathbf{4}, 5$ and 3], required the ANR-property. We prove that the following spaces are absolute retracts: (1) every complete metric linear space $(=F$-space) with a Schauder basis, (2) certain function spaces which include $L_{p}(p \geqslant 0)$ and Orlicz spaces, and (3) additive subgroups consisting of all integer-valued functions in certain function spaces. Consequently, each of these spaces, when complete and separable, is homeomorphic to a Hilbert space $[4,5]$. The argument used in verifying the AR-property of the above examples is also employed to show that the $\mathrm{AR}$ and the $\mathrm{AE}(\mathscr{C})$ (absolute extension property for compacta) properties coincide for $\sigma$-compact convex sets. This enables us to find a dense convex topological copy of $\Sigma$, the linear span of the Hilbert cube in the Hilbert space $l_{2}$, in every separable infinite-dimensional complete convex set.

Our approach is very elementary and mostly involves the natural equiconnected structures of convex sets and contractible groups. We also employ the admissibility notion introduced by Klee $[\mathbf{9}, 10]$.

2. Equiconnected spaces that are ANRs. A space $X$ is said to be locally equiconnected if there exists a map $c: V \times[0,1] \rightarrow X$, where $V$ is a neighborhood of the diagonal in $X \times X$, such that $c\left(x_{1}, x_{2}, 0\right)=x_{1}, c\left(x_{1}, x_{2}, 1\right)=x_{2}$, and $c(x, x, t)=x$ for every $x_{1}, x_{2}, x \in X$ and $t \in[0,1]$. The map $c$ is called a local equiconnecting function. The space $X$ is an equiconnected space, and $c$ is an equiconnecting function if $c$ is defined on $X \times X \times[0,1]$. In the sequel we will be interested in the following two examples of locally equiconnected spaces.

Received by the editors February 6, 1982 and, in revised form, May 22, 1984.

1980 Mathematics Subject Classification. Primary 54C55, 52A07: Secondary 54D45, 57N17.

Key words and phrases. Convex sets, locally contractible groups, ANR spaces. ANE spaces. 
EXAmPle 1. A convex subset of a metric linear space is an equiconnected space with a naturally defined equiconnecting function $c\left(x_{1}, x_{2}, t\right)=(1-t) x_{1}+t x_{2}$.

EXAmPLE 2. A (locally) contractible topological group is a (locally) equiconnected space. A (local) equiconnecting function can be defined by

$$
c\left(g_{1}, g_{2}, t\right)=g_{2} \circ(h(e, t))^{-1} \circ h\left(g_{2}^{-1} \circ g_{1}, t\right),
$$

where $h$ is a homotopy with $h(g, 0)=g$ and $h(g, 1)=e$ for all $g$ (a consequence: contractible topological groups are also locally contractible).

The following slight generalization of a theorem of Hanner [6] will be our main tool in applications.

THEOREM 1. Let $X$ be a locally equiconnected metric space. If $\mathrm{id}_{X}=\lim _{\mathrm{co}}\left(\beta_{n} \circ \alpha_{n}\right)$, where $\alpha_{n}$ is a map of $X$ into some $X_{n} \in A N R$ and $\beta_{n}$ is a map of $X_{n}$ into $X$, $n=1,2, \ldots$, then $X \in A N$. $^{1}$

Theorem 1 can easily be derived from the following

Lemma 1. Let $A$ be a closed subset of a metric space $(Z, \rho)$ and let $(X, d)$ be a locally equiconnected space. Suppose $f: A \rightarrow X$ is a map such that $f=\lim _{\mathrm{co}} f_{n}$, where each map $f_{n}$ extends to a neighborhood of $A$. Then $f$ extends to a neighborhood of $A$.

Proof. Let $\left\{U_{n}\right\}$ be a sequence of open neighborhoods of $A$ such that, for each $n$, $f_{n}$ extends to $U_{n}, \operatorname{cl}\left(U_{n+1}\right) \subset U_{n}$, and $\bigcap_{1}^{\infty} U_{n}=A$. For each $z \in Z$ pick $a_{z} \in A$ such that $\rho\left(z, a_{z}\right) \leqslant 2 \rho(z, A)$. We may assume each $U_{n}$ is small enough so that for each $z \in U_{n}, d\left(f_{n}(z), f_{n}\left(a_{z}\right)\right) \leqslant 1 / n$. Let $\lambda: U_{1} \backslash A \rightarrow(1, \infty)$ be a map such that for each $n, \lambda\left(U_{n} \backslash \operatorname{cl}\left(U_{n+1}\right)\right) \subset(n, n+1)$ and $\lambda\left(\operatorname{bd}\left(U_{n+1}\right)\right)=n+1$. Let $c: V \rightarrow X$ be a local equiconnecting function defined on a neighborhood of the diagonal in $X \times X$. For each $n \geqslant 2$ set $V_{n}=\left\{z \in U_{n}:\left(f_{n-1}(z), f_{n}(z)\right) \in V\right\}$.

Consider $W=\cup_{n=2}^{\infty}\left(V_{n} \backslash U_{n+1}\right) \cup A$. We claim that $W$ is a neighborhood of $A$ and $f$ extends over $W$. The hypothesis $f=\lim _{\text {co }} f_{n}$ means that, for each $a \in A$ and $\varepsilon>0$, there exists a neighborhood $N(a)$ in $A$ and an integer $N$ such that for each $a^{\prime} \in N(a)$ and $n \geqslant N, d\left(f_{n}\left(a^{\prime}\right), f_{n}(a)\right)<\varepsilon$. Together with the above condition on each $U_{n}$, this implies the following:

(1) For each $a \in A$ and $\varepsilon>0$, there exists a neighborhood $U(a)$ in $Z$ and an integer $N$ such that for each $n \geqslant N$ and $z \in U(a) \cap U_{n}, d\left(f_{n}(z), f(a)\right)<\varepsilon$.

Thus for $U(a)$ small enough, we have $U(a) \cap U_{n} \subset V_{n}$ for all large $n$, hence $U(a) \cap U_{n} \subset W$. An extension $\bar{f}: W \rightarrow X$ is defined by the formula

$$
\bar{f}(a)= \begin{cases}c\left(f_{n-1}(z), f_{n}(z), \lambda(z)-n\right), & z \in V_{n} \backslash U_{n+1}, \\ f(z), & z \in A . \square\end{cases}
$$

\section{Applications.}

THEOREM 2. Every F-space $E$ with a Schauder basis (more generally: every F-space admitting a sequence $T_{n}: E \rightarrow E$ of finite rank continuous linear operators such that $T_{n} x \rightarrow x$ for every $x \in E$ ) is homeomorphic to a Hilbert space.

$\operatorname{id}_{X}=\lim _{\mathrm{co}}\left(\beta_{n} \circ \alpha_{n}\right)$ means that id ${ }_{X}$, the identity on $X$, is the limit in the compact-open topology of the sequence $\left\{\beta_{n} \circ \alpha_{n}\right\}$. 
Proof. The Banach-Steinhaus Theorem yields $\lim _{\text {co }} T_{n}=\mathrm{id}_{E}$. Since $T_{n}(E)$ is isomorphic to a Euclidean space, it is an AR. Applying Theorem 1, we obtain $E \in \mathrm{AR}$, which implies [4] that $E$ is homeomorphic to a Hilbert space.

The next example requires a preliminary description. Let $(S, \Sigma, \mu)$ be a $\sigma$-finite measure space. A function space is a metric linear space $(X,\|\cdot\|)$ of $\mu$-equivalence classes of real $\Sigma$-measurable functions on $S$ satisfying

(i) if $|f(s)| \leqslant|g(s)|$ a.e. and $g \in X$, then $f \in X$ and $\||f|\| \leqslant\||g|\|$,

(ii) for every $\left\{f_{n}\right\} \subset X,\left|f_{n}(s)\right| \leqslant|f(s)|$ a.e. for $n=1,2, \ldots$, with $0 \leqslant f \in X$, we have $\left\|f_{n}\right\| \rightarrow 0$ iff $f_{n} \rightarrow 0$ in measure on every set of $\mu$-finite measure (cf. [8]).

Function spaces which are Banach spaces were intensively investigated in a series of papers by Luxemburg and Zaanen (see, e.g., [12]). In our setting we permit function spaces to be nonlocally convex $(\|\cdot\|$ is not supposed to be homogeneous; we only require $(f, g) \rightarrow\|f-g\|$ to be a metric on $X)$.

THEOREM 3. Every separable function space $X$ is an absolute retract.

Proof. First, we construct a set $E=\bigcup_{1}^{\infty} E_{n}, E_{n} \in \Sigma$ for $n=1,2, \ldots$, with the properties:

(1) $\chi_{E} \cdot f=f$ for every $f \in X$,

(2) $\chi_{E_{n}} \in X$, and

(3) $\mu\left(E_{n}\right)<\infty$ for $n=1,2, \ldots$

To this end pick a dense subset $\left\{f_{n}\right\}_{1}^{\infty}$ of $X$. Write $E=\bigcup_{1}^{\infty}\left\{s: f_{n}(s) \neq 0\right\}$. Given $f \in X$ and $\varepsilon>0$, there exists $f_{n}$ with $\left\|f-f_{n}\right\| \leqslant \varepsilon$. Using condition (i) we estimate

$$
\left\|f-\chi_{E} \cdot f\right\| \leqslant\left\|f-f_{n}\right\|+\left\|f_{n}-\chi_{E} f\right\| \leqslant 2 \cdot \varepsilon
$$

this shows (1). Now, noting that

$$
\left\{s: f_{n}(s) \neq 0\right\}=\bigcup_{1}^{\infty}\left\{s:\left|f_{n}(s)\right| \geqslant k^{-1}\right\},
$$

it is easy to determine $E_{n}(n=1,2, \ldots)$ in order to fulfill (2) and (3).

We apply Theorem 1 , by letting $\alpha_{n}(f)=\chi_{E_{n}} \cdot \sup (\inf (n, f),-n)$ and $X_{n}=$ $\left\{f \in X: \chi_{E_{n}} \cdot f=f\right.$ and $|f(s)| \leqslant n$ a.e. $\}$. Clearly, by (2) and (i), $\alpha_{n}(X) \subset X_{n}$. It is elementary to show that $\left|\left(\alpha_{n}(f)-\alpha_{n}(g)\right)(s)\right| \leqslant|f(s)-g(s)|$ a.e. and $\left|\left(\alpha_{n+1}(f)-f\right)(s)\right| \leqslant\left|\left(\alpha_{n}(f)-f\right)(s)\right|$ a.e. for every $f, g \in X$ and $n=1,2, \ldots$ This, in turn, guarantees the equiuniform continuity of $\left\{\alpha_{n}\right\}$ by (i), and $\left\|\alpha_{n}(f)-f\right\| \rightarrow 0$ by (ii) and (1). Now, the fact that $\lim _{\mathrm{co}} \alpha_{n}=\mathrm{id}_{X}$ easily follows. Moreover, observe that $X_{n}$ is a convex subset of $X$ satisfying: $\left\|f_{n}-f_{0}\right\| \rightarrow 0$ iff $f_{n} \rightarrow f_{0}$ in measure iff $f_{n} \rightarrow f_{0}$ in $L_{1}\left(E_{n}, \mu\right)$ for $\left\{f_{n}\right\}_{n=0}^{\infty} \subset X_{n}$. Thus $X_{n}$ is affinely homeomorphic to a convex subset of $L_{1}$. Finally, the Borsuk-Dugundji Theorem (see [2, p. 58]) ensures $X_{n} \in \mathrm{AR}$ and Theorem 1 is applicable.

Note 1. The separability condition in Theorem 3 may be dropped, assuming, additionally, $\chi_{s} \in X$.

Note 2. The same proof yields $\left\{f \in X:|f(s)| \leqslant\left|f_{0}(s)\right|\right.$ a.e. $\} \in \mathrm{AR}$, where $f_{0}$ is any $\Sigma$-measurable nonnegative function. 
Consider the subset $G$ of $L_{2}(0,1)$ consisting of all integer-valued functions. $G$ occurs as a closed additive subgroup of $L_{2}(0,1)$ admitting no one-parameter subgroups. Answering a question of Wojtynski, we describe the topological nature of G.

THEOREM 4. Let $X$ be a separable function space defined on a $\sigma$-finite measure space $(S, \Sigma, \mu)$ with atomless measure $\mu$. The additive subgroup $G$ of $X$ consisting of all integer-valued functions is an AR. Moreover, if $G$ is complete, then $G$ is homeomorphic to $l_{2}$.

Proof. We will follow the proof of Theorem 3. Having constructed sets $E$ and $E_{n}$ $(n=1,2, \ldots)$, we consider $\alpha_{n}(f)=\chi_{E_{n}} \cdot \sup (\inf (n, f),-n)$ for $f \in G$. Clearly $\alpha_{n}(G) \subset\left\{f \in G: \chi_{E_{n}} \cdot f=f\right.$ and $|f(s)| \leqslant n$ a.e. $\}=G_{n}$. The argument used in Theorem 3 shows that $\lim _{\mathrm{co}} \alpha_{n}=\mathrm{id}_{G}$, and the topology on $G_{n}$ coincides with the topology of convergence in measure. Hence, by a theorem of Bessaga and Pelczyński (see [2, p. 201]) the set $G_{n}$ is homeomorphic to $l_{2}$; so $G_{n} \in \mathrm{AR}$.

Now, in order to apply Theorem 1 we will show that $G$ is contractible (cf. Example 2). Since $\mu$ is $\sigma$-finite and atomless, there exists a continuous map $\lambda$ : $[0,1] \rightarrow(\Sigma, d)$, where $d\left(E_{1}, E_{2}\right)=\mu\left(E_{2} \backslash E_{1}\right)+\mu\left(E_{1} \backslash E_{2}\right)$, such that $\lambda(1)=\varnothing$, $\lambda(0)=S$ and $\mu\left(\bigcup_{\varepsilon \geqslant \varepsilon_{0}} \lambda(\varepsilon)\right)<\infty$ for every $\varepsilon_{0}>0$. Write $h(f, t)=\chi_{\lambda(t)} \cdot f$ and note that $h(f, 0)=f$ and $h(f, 1)=0$ for every $f \in G$. If $\left\|f_{n}-f\right\| \rightarrow 0$ and $t_{n} \rightarrow t$, then by (i),

$$
\begin{aligned}
\left\|h\left(f_{n}, t_{n}\right)-h(f, t)\right\| & \leqslant\left\|\chi_{\lambda\left(t_{n}\right)} \cdot f_{n}-\chi_{\lambda\left(t_{n}\right)} \cdot f\right\|+\left\|\chi_{\lambda\left(t_{n}\right)} \cdot f-\chi_{\lambda(t)} \cdot f\right\| \\
& \leqslant\left\|f_{n}-f\right\|+\left\|\chi_{\lambda\left(t_{n}\right)} \cdot f-\chi_{\lambda(t)} \cdot f\right\| .
\end{aligned}
$$

Since $\chi_{\lambda\left(t_{n}\right)} \cdot f-\chi_{\lambda(t)} \cdot f \rightarrow 0$ in measure, the continuity of $h$ follows from (ii). The last part of Theorem 4 follows from [5].

4. Admissibility and $\mathbf{A R}$ properties. Assume $C$ is a convex subset of a metric linear space $(E,\|\cdot\|)$. Following Klee [9], we say that $C$ is admissible if for every compact set $K \subset C$ the identity map id ${ }_{K}$ can be uniformly approximated by maps $\phi: K \rightarrow C$ with $\operatorname{dim} \operatorname{span}(\phi(K))<\infty$. Klee [9] proved that admissible $F$-spaces are $\mathrm{AE}(\mathscr{C})$. The following theorem extends the result of Klee.

THEOREM 5. A convex set $C$ is an $A R$ iff $\mathrm{id}_{C}=\lim _{\mathrm{co}} \phi_{n}$, where each $\phi_{n}$ is a locally finite-dimensional (l.f.d.) map, i.e., every point of $C$ has a neighborhood $U$ with $\operatorname{dim} \operatorname{span}\left(\phi_{n}(U)\right)<\infty$.

We will employ the following

Lemma 2. Let $\left\{A_{n}\right\}$ be a countable closed cover of a metric space $A$ and $C$ a convex subset of a metric linear space. Suppose $f: A \rightarrow C$ is a map such that for each $n, f \mid A_{n}$ is the uniform limit of l.f.d. maps. Then $f$ is the uniform limit of l.f.d. maps.

Proof of Lemma 2. Fix a map $f: A \rightarrow C$ and $\varepsilon>0$. Choose an 1.f.d. map $f_{n}$ : $A_{n} \rightarrow C$ with $\left\|f_{n}-f \mid A_{n}\right\|<2^{-n} \varepsilon$. Since finite-dimensional convex subsets of $C$ are ARs, applying a partition of unity argument, we construct l.f.d. maps $\bar{f}_{n}: A \rightarrow C$ 
such that $\bar{f} \mid A_{n}=f_{n}$. There exists a locally finite partition of unity $\left\{\lambda_{n}\right\}$ with $\lambda_{n}^{-1}((0,1]) \subset\left\{a \in A:\left\|\bar{f}_{n}(a)-f(a)\right\|<2^{-n} \varepsilon\right\}$. Letting $\bar{f}(a)=\sum_{1}^{\infty} \lambda_{n}(a) \bar{f}_{n}(a)$, we see that $\bar{f}$ is an l.f.d. map satisfying

$$
\|f(a)-\bar{f}(a)\| \leqslant \sum_{1}^{\infty}\left\|\lambda_{n}(a)\left(f(a)-\bar{f}_{n}(a)\right)\right\| \leqslant \sum_{1}^{\infty} 2^{-n} \varepsilon=\varepsilon .
$$

Proof of Theorem 5. Necessity. Since $C \in \mathrm{AR}$, for every $\varepsilon>0$ there exists a locally finite-dimensional metric simplicial complex $K$ and maps $\phi: C \rightarrow|K|, \psi$ : $|K| \rightarrow C$ such that $\|\psi \phi(x)-x\|<\varepsilon$. Clearly $|K|$ is a countable union of closed finite-dimensional sets. It can be easily verified that every map of a finite-dimensional metric space into $C$ is the uniform limit of l.f.d. maps (cf. [4]). Thus, by Lemma 2, the map $\psi$ is the uniform limit of l.f.d. maps. Consequently, $i_{C}$ is also the uniform limit of l.f.d. maps.

Sufficiency. Let $f: A \rightarrow C$ be a map, where $A$ is a closed subset of a inetric space $Z$. Using a partition of unity argument and the fact that finite-dimensional convex subsets of $C$ are ARs, we construct maps $f_{n}: Z \rightarrow C$ such that $f_{n} \mid A=\phi_{n} \circ f$ for $n=1,2, \ldots$. Since $\lim _{\text {co }} f_{n}=f$, Lemma 1 ensures that $f$ extends to a neighborhood of $A$. Finally, since $C$ is a contractible ANR, it is an AR.

Note 3. The same proof (for the sufficiency part) yields $C \in \mathrm{AE}(\mathscr{C})$ iff $C$ is admissible.

Note 4. Applying Lemma 2 in the sufficiency part of the proof of Theorem 5, one can show that $C \in \mathrm{AR}$, provided that $\mathrm{id}_{C}=\lim _{\text {co }} \phi_{n}$ such that each $\phi_{n}(C)$ is a countable union of closed finite-dimensional sets (cf. [1]). More generally, one may only require that each $\phi_{n}(C)$ is a $C$-space (see [7]). This follows from the fact that Lemma 2 holds when $A$ is a subspace of $C$ with the weaker assumption that each $A_{n}$ is a (not necessarily closed) $C$-space.

COROLlaRY 1. For a $\sigma$-compact convex set the following conditions are equivalent:

(a) $C \in \mathrm{AR}$,

(b) $C=\cup_{1}^{\infty} A_{n}$ with $A_{n}=\operatorname{cl}\left(A_{n}\right) \in \mathrm{AE}(\mathscr{C})$ for $n=1,2, \ldots$,

(c) $C$ is admissible.

Proof. This is a consequence of Theorem 5 and Lemma 2.

COROllary 2. Every separable infinite-dimensional complete convex set $C$ contains a dense convex subset $\tilde{C}$ homeomorphic to $\Sigma=\left\{\left(x_{n}\right) \in l_{2}: \sum_{1}^{\infty}\left(n x_{n}\right)^{2}<\infty\right\}$.

Proof. The proof of [3, Proposition 3.4] shows that there are affine copies $C_{1}, C_{2}, \ldots$ of compact convex subsets of $l_{2}$ with $\tilde{C}=\cup_{1}^{\infty} C_{n}$ dense in $C$ and such that $\left\{C_{n}\right\}$ is a so-called strong universal tower for compacta. By Corollary 1 we have $\tilde{C} \in \mathrm{AR}$. Finally, the assertion follows from [3, Theorem 4.1].

5. A generalization. The problem of whether a separable metric space $X \in$ $\operatorname{ANE}(\mathscr{C})$ is an ANR, posed by Kuratowski [11], remains unsolved. Apparently, an answer to this problem is not known even for $\sigma$-compact $X$. Our consideration leads to a partial answer. 
Proposition. Let $(X, d)$ be a o-compact space and $c$ a local equiconnecting function defined for all $x_{1}, x_{2}$ with $d\left(x_{1}, x_{2}\right)<\varepsilon$ for some $\varepsilon>0$ and satisfying$$
d\left(c\left(x_{1}, x_{2}, t\right), x_{2}\right) \leqslant d\left(x_{1}, x_{2}\right) \text { for every } x_{1}, x_{2} \in X \text { and } 0 \leqslant t \leqslant 1 .
$$

If $X=\cup_{1}^{\infty} X_{n}$ and each $X_{n}=\operatorname{cl}\left(X_{n}\right) \in \operatorname{ANE}(\mathscr{C})$, then $X \in \operatorname{ANR}$.

Proof (Sketch). First, to show that $X \in \operatorname{ANE}(\mathscr{C})$, consider a map $f: A \rightarrow X$, where $A$ is a closed subset of a compact set $Z$. Imitating the argument of Lemma 2 with $A_{n}=f^{-1}\left(X_{n}\right)$, one can show that $f$ is a uniform limit of maps defined on neighborhoods of $A$ in $Z$. Lemma 1 guarantees a neighborhood extension of $f$. To repeat the same argument in the case where $A$ is a closed $\sigma$-compact subset of a metric space, we use the fact that any map of a compact subset $B$ of a metric space $Y$ into an $\mathrm{ANE}(\mathscr{C})$ extends to a neighborhood of $B$ in $Y$.

Note 5. If $X$ is a convex subset of a metric linear space $(E,\|\cdot\|)$, then the equiconnecting function of Example 1 satisfies (*) with respect to the metric $d$ induced by $\|\cdot\|$. If $X=(G, d)$ is a locally contractible topological group and $d$ is left-invariant, then the local equiconnecting function of Example 2 satisfies (*), provided that

$(* *) \quad d(h(g, t), h(e, t)) \leqslant d(g, e)$ for every $g \in G$ and $0 \leqslant t \leqslant 1$.

The author wishes to thank Doug Curtis for his careful reading of the paper and several helpful comments. The proof of Lemma 1, more direct than the original one, is due to him.

\section{REFERENCES}

1. C. Bessaga and T. Dobrowolski, Some problems in the border of functional analysis and topology, Proc. Internat. Conf. Geometric Topology (Warsaw, 1978), PWN, Warsaw, 1980, pp. 39-42.

2. C. Bessaga and A. Pelczyński, Selected topics in infinite-dimensional topology, PWN, Warsaw, 1975.

3. D. W. Curtis, T. Dobrowolski and J. Mogilski, Some applications of the topological characterizations of the sigma-compact spaces If and $\Sigma$, Trans. Amer. Math. Soc. 284 (1984), 837-846.

4. T. Dorowolski and H. Torunczyk, On metric linear spaces homeomorphic to $I_{2}$ and convex sets homeomorphic to Q, Bull. Acad. Polon. Sci. Sér. Sci. Math. Astronom. Phys. 27 (1979), 883-887.

5. Separable complete ANR's admitting group structures are Hilbert manifolds, Topology Appl. 12 (1981), 229-235.

6. O. Hanner, Some theorems on absolute retracts, Ark. Mat. 1 (1951), 389-408.

7. W. E. Haver, A covering property for metric spaces, VPI Topology Conf. (R. F. Dickman and P. Fletcher, eds.), Lecture Notes in Math., vol. 375, Springer-Verlag, New York, 1974, pp. 108-113.

8. J. Ishii, On the admissibility of function spaces, J. Fac. Sci. Hokkaido Univ. 19 (1965), 49-55.

9. V. L. Klee, Shrinkable neighborhoods in Hausdorff linear spaces, Math. Ann. 141 (1960), 281-285.

10. , Leray-Schauder theory without local convexity, Math. Ann. 141 (1960), 286-296.

11. K. Kuratowski, Sur quelques problemes topologiques concernant le prolongement des fonctions continues, Colloq. Math. 2 (1951), 186-191.

12. W. A. J. Luxemburg and A. C. Zaanen, Notes on Banach function spaces, Indag. Math. 25 (1963), $135-147$.

Institute of Mathematics, Warsaw University, 00 - 901 Warsaw, PKin, IXP, Poland

Department of Mathematics, University of Alabama, University, Alabama 35486

Current address: Department of Mathematics, Texas A \& M University, College Station, Texas 77843 